\title{
DRUG-RESISTANT TUBERCULOSIS: RECENT APPROACH IN POLYMER BASED NANOMEDICINE
}

\author{
JESSY SHAJI ${ }^{*}$, MAHMOOD SHAIKH ${ }^{\mathrm{a}}$
}

\begin{abstract}
aDepartment of Pharmaceutics, Prin. K. M. Kundnani College of Pharmacy, 23, Jote Joy Building, Rambhau Salgaonkar Marg, Cuffe Parade, Mumbai 400005, India
\end{abstract}

Email: jessy.shaji@gmail.com

Received: 17 Feb 2015 Revised and Accepted: 12 Aug 2016

\begin{abstract}
Tuberculosis (TB) had been a leading chronic bacterial infection since decades. Current therapeutic management of Mycobacterium tuberculosis (MTB) is inadequate due to the lengthy course of treatment, drug-related side effects and ill-planned therapy, and these factors can lead to therapeutic failure and the emergence of drug-resistant TB. The Multi-drug-resistant (MDR) TB needs a lengthy course of treatment with secondline antitubercular drugs (ATDs) having higher side effects and cost. The misuse of second-line ATDs may result in extremely drug-resistant (XDR) strain which is very difficult to treat and require high doses of drugs resulting in more toxicity and side effects. This review highlights the need for novel drug delivery for the treatment of drug-susceptible and resistant TB. The characteristics of the nanoparticulate system in ATDs delivery and its approach in the MDR and XDR TB are discussed. The lung is the site of infection in pulmonary TB and the targeted drug delivery to the site of infection helps in achieving increased efficacy with less dose further reducing the side effects and toxicity. The symbiotic association of nanotechnology and pulmonary drug delivery give rise to an efficient inhalable polymer based nanoparticulate system containing ATDs for the better management of drug-susceptible and resistant TB. Various ATDs loaded polymer based nanocarrier systems like Alginate, PLGA, Chitosan and Gelatin nanocarriers are discussed in detail. Thus, this review highlights the current research in pulmonary drug delivery of polymer based ATDs nanomedicine and their importance in control of drug-resistant TB.
\end{abstract}

Keywords: Tuberculosis, Drug resistant, Nanotechnology, Nanocarriers, Polymer, Pulmonary

(C) 2016 The Authors. Published by Innovare Academic Sciences Pvt Ltd. This is an open access article under the CC BY license (http://creativecommons. org/licenses/by/4. 0/) DOI: http://dx.doi.org/10.22159/ijpps.2016v8i10.11295

\section{INTRODUCTION}

Tuberculosis (TB) is a chronic infectious disease, and the latent TB infection has affected over one-third of the people on this planet. After the Human immunodeficiency virus (HIV), the Mycobacterium Tuberculosis (MTB) is considered as a single pathogen responsible for the foremost cause of mortality [1]. This infection affected the people of both the developing and the developed countries [2]. The prevalence of the TB still exists despite the five decades of various treatment programs with the presence of the effective drugs for this disease. The effective and preventive measures should be taken by concerned bodies and authorities around the world as the number of people being infected and dying due to the TB infection is alarming in this decade [3]. Moreover, the co-occurrence of the HIV and the resistant TB infection has been a challenge for the control of TB across the globe [4].

Management of Multi-drug resistant tuberculosis (MDR-TB) with conventional drug delivery system has seen an increase in drug resistance. This resistance has resulted from poor patient compliance due to the drug-related side effects from high doses, the frequency of dosing and lengthy treatment. Moreover, the drawbacks like inefficient efficacy, poor tolerability of second line ATDs leads to withdrawal from treatment cause further resistance like extremely drug-resistant tuberculosis (XDR-TB) which results in the increase in high death rate due to the TB infection [5]. This indicates the need for new drug delivery technology such as the use of novel controlled release nanoparticulate system like polymeric nanocarrier systems containing existing Anti-tubercular drugs (ATDs). These nanocarriers will help in overcoming the problems associated with the conventional drug delivery system in the treatment of drug susceptible and resistant TB [6]. Nanocarrier systems can be used to deliver drugs through the parenteral, oral, nasal and pulmonary route. Moreover, Pulmonary drug delivery (PDD) of these ATDs loaded nanocarriers helps in depositing sitespecific drugs at high concentrations within the diseased lungs, thereby reducing the overall dose of a drug to patients which in turn reduces systemic side effects [7-12]. Thus, the ATDs loaded sustained release nanocarriers together with site-specific targeting holds the potential to treat intermittent therapy of drug susceptible and resistant TB. The relevant literature on this review was collected by searching PubMed and Google Scholar database. The Keywords used for the searches were "Tuberculosis", "Drugresistant", "Nanotechnology", "Nanocarriers", "Polymeric nanoparticles", "Pulmonary drug delivery", "Polymer-based nanomedicines, etc. The range of years selected was from 1970 to present. The literature was thoroughly analyzed and data generated as per the relevant topic of this review.

\section{Transmission of TB disease, pathogenesis, and its types}

TB is an airborne disease transmitted by MTB from diseased persons to normal persons. MTB is anaerobic, non-spore-forming, nonmotile rod-shaped bacteria which is highly resistant to alcohol and acid. The infection transmitted by diseased person's form droplets in the air during speaking, coughing and sneezing and the pulmonary TB consist of $80 \%$ of overall cases of TB infection [13] TB is generally classified as latent or active TB [14]. Various researchers like Armstrong and Hart [15], Aderem and Underhill [16], Pethe et al. [17] and Krishnan et al. [18] had explained the TB pathogenesis in detail. The three types of a pathogenic strain of TB are Drug-susceptible, MDR, and XDR strain. The Drug-susceptible TB strain is treated by first line drugs namely Ethambutol (EMB), Isoniazid (INH), Pyrazinamide (PZA), and Rifampicin (RIF). The strain of MDR-TB which is resistant to the INH and RIF are difficult to be managed. The XDR-TB develops when the microbial strain is resistant to the first line drugs namely INH and RIF and also to the second line drugs like some Fluoroquinolones and injectable drugs. Further resistant in XDR strain possesses a major challenge in the treatment of TB.

Conventional chemotherapy of TB, its limitations and emergence of drug resistance

Existed ATDs include ten US FDA approved drugs, and the first-line ATDs are the first choice in the Drug-susceptible-TB therapy due to their high efficacy and least side effects. The Drug-susceptible-TB therapy consists of intensive and continuation phase, the first phase consist of the first-line ATDs such as EMB, INH, PZA and RIF for the 
period of four months while the latter phase lasts for four to six months $[19,20]$. In India, available TB treatment is performed under the scheme of directly observed therapy known as DOTS.

The assumption of being cured and discontinuation of the therapy prematurely creates a preferable condition for drug resistance [21] and also the ill-planned monotherapy causes the development of a resistant strain of bacteria against the first-line ATDs. [19, 20]. The problems of overdose or under-dosing related to the monotherapy can be prevented by using the combination therapy [20]. The Fixed dose combination (FDC) product $[19,22]$ contains a combination of first-line ATDs. The quality of drugs in FDC product affects the treatment of TB disease. The mismanagement or misuse of such drugs in FDC products lead to the MDR strain of bacteria which required second-line ATDs. The limitations of conventional therapy of TB and causes of drug resistance is shown in fig. 1.
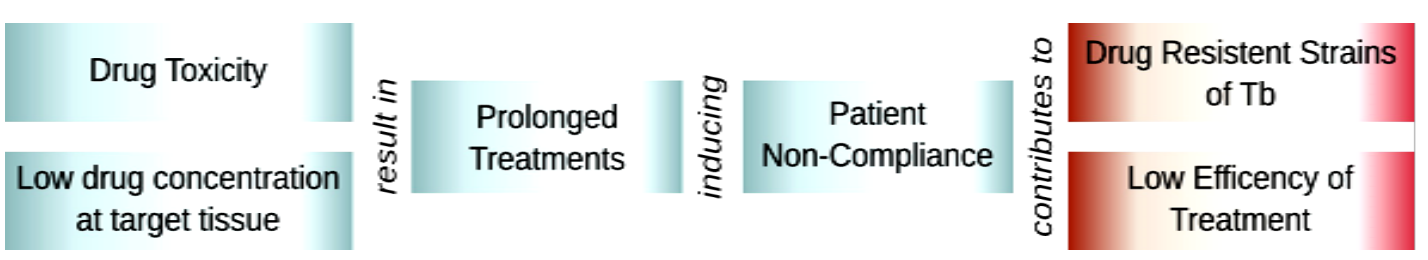

Fig. 1: Drawbacks of conventional TB therapy and emergence of drug resistant [23]

Recently there is a rise in the resistant strain, and this has resulted in the urgent need of a strategy and planning in all respective medical, healthcare and pharmaceutical field for combating this crisis [4]. WHO report on "XDR-TB: Extensively Drug-resistant Tuberculosis" in the year 2007, stated that the resistant strain of MTB and, the negligence in the proper implementation of a regimen involving first line drugs are the reasons responsible for the MDRTB. Other reasons like poor planning by the authorities and the government may also result in the emergence of resistant strain [24]. The option for treating the resistant strains like MDR and XDR strains are very limited. The MDR-TB treatment therapy is lengthy and last up to $2 \mathrm{y}$; sometimes such lengthy therapy results in patient withdrawal from the course due to adverse effect and causes further severity in the disease. Such patient noncompliance results in the increase in high death rate due to the TB disease [5]. The development of drug-resistant may be due to the misuse of ATDs and also the ill-planned therapy [25]. The misuse of second line ATDs causes further resistant strain of bacteria [26, 27] causing MDR-TB and this strain may develop further resistant and can cause XDR-TB [28] and even total drug-resistant (TDR) TB which is difficult to treat [29] and require very high doses of drugs resulting in more toxicity and side effects. A future epidemic of this disease with a lethal XDR-TB and TDR-TB strain having very limited effectiveness for treatment can jeopardize the development in control of this disease till date. Moreover, the inadequate infrastructure in many countries gives rise to the inaccurate diagnosis of this resistant strains causing a further increase in mortality especially in a case of infection with the HIV disease. The resistant strains are responsible for $10 \%$ of deaths from overall deaths due to TB infection. Countries like India and China are having most cases of MDR-TB globally.

\section{Need for new drug delivery system (NDDS)}

The development of controlled release system which negates the administration of ATDs regularly will be helpful in overcoming patient noncompliance and avoiding the limitations related to the current conventional chemotherapy. The treatment for drugresistant $\mathrm{TB}$ was found to be inadequate in a case where the susceptible drug patient was already administered with second line ATDs [2]. The slow development of novel therapies for TB treatment and the use of five decades old ATDs till date have made the researchers to put a highlight on the demand of novel strategy for combating the MDR and XDR strains of TB [4]. The new candidates showing susceptibility against the TB strains including resistant strains are in research and development, few are in clinical trials, and many existing drugs are also revaluated for their efficacy against the TB [30]. Recently the USFDA approved a new drug Bedaquiline to be used in combination with other ATDs when there is no other drug available to treat MDR-TB. The Bedaquiline had shown the cases of heart failure which may result in its removal from market and also this drug have not passed through the phase III trial. The finding of new drugs having activity against the drug resistance TB strain is on priority. But the urgent need demands the new drug delivery technology such as the use of novel controlled release nanoparticulate system containing the existing ATDs may help in combating the TB disease.

\section{Nanoparticulate system as an interesting NDDS for TB}

A nanoparticulate system composed of biodegradable polymers and loaded with ATDs can overcome drawbacks associated with conventional therapy and show some novel and significant characteristics. The sustained release of a drug is observed with gradual breaking down of nanoparticles (NPs). The initial sharp peak associated with the traditional drug delivery system is avoided in case of a nanoparticulate system which shows the more constant drug concentration and longer time of residence in the body. This characteristic helps in efficient as well as less toxic drug delivery to the diseased person. The encapsulated drugs in nanoparticulate system showed efficient results in combating the infection at what would normally be sub-therapeutic doses of a drug. [31, 32].

The drug concentration from NPs doesn't drop from the certain threshold which helps in overcoming the drug resistance. The nanoparticulate system can be orally administered and also by the pulmonary route which shows significant results in treating TB [33]. The macrophages take up the particles in a range of $200 \mathrm{~nm}$ to $6 \mu \mathrm{m}$ selectively via a process of phagocytosis.

\section{Nanotechnological approach in MDR-TB and XDR-TB}

The increase in the occurrence of MDR-TB with its deadly association with HIV has necessitated the use of nanotechnological approach like the encapsulation of second line ATDs. Various existing first and second line ATDs can be incorporated into nanocarrier systems by using nanotechnological approach [34]. The second line ATD Ethionamide was made into NPs by encapsulation technology and evaluated, but no in vivo studies is reported on this formulation [35]. After a decade, various researchers had loaded Ethionamide into Poly (lactide-coglycolide) acid (PLGA) nanocarriers and characterized [6]. Also, the Fluoroquinolones and some azole antifungals were formulated by nanoencapsulation methods and showed desirable properties $[36,37]$.

The Azole antifungals like Econazole and Clotrimazole had shown potent anti-TB activity against drug-susceptible [38, 39], drugresistant and latent TB [40]. The nanoencapsulation method had been used for the formulation of Streptomycin intended for oral administration. Streptomycin is among the cost effective drugs utilize in specific cases such as in withdrawal of RIF and INH, treatment failure or relapse, TB meningitis, some cases of MDR-TB and co-treatment with anti-HIV drugs. The PLGA nanocarriers containing Streptomycin was not only given by oral route but also, they possess various merits such as the increase in therapeutic efficacy, bioavailability and absence of nephrotoxicity as opposed to the free form of the drug [41]. These studies showed that the polymeric nanocarriers possess significant characteristics to be used as a carrier system for second line ATDs. But the variability of drug-resistant with respect to the individual treatment is responsible for the limited data on nanotechnological approach in drug-resistant TB. 


\section{Need of PDD of NPs containing ATDs}

The NPs are found to be promising candidates in PDD [42] as their size can modulate with respect to different formulation methods and parameters. The variability in particle size helps to target different sites in the lungs. Moreover, the symbiotic association of nanotechnology with the PDD helps in targeting the ATDs loaded nanocarriers to the site of infection i.e. AMs. Moreover, the NPs can be engineered by attaching different ligands for active targeting of an area of interest like alveolar macrophages (AMs) [43]. Improved bioavailability is achieved with less dose as compared to other drug delivery system [44]. Thus, efficient therapy is achieved with reduced side effects and toxicity [45]. For PDD of ATDs various nanocarrier systems as Vesicle-based, lipid based, emulsion based, and polymer based are formulated and tested [46]. Until now, no nanoformulation of ATDs intended for PDD has been in the market. There have been several studies undertaken for targeting the ATDs directly to the infection site enhancing the local therapeutic effect and also to decrease the systemic exposure of drug doses [7-12].

\section{Merits and challenges in PDD of NPs containing ATDs}

Lungs are the infection site in pulmonary ТВ $[47,48]$ and therefore, the pulmonary route can be a promising in drug delivery to the infection site [49-51]. The targeted delivery to the Lungs will give an increased ATDs efficacy with less dose and also have reduced side effects and toxicity [52].

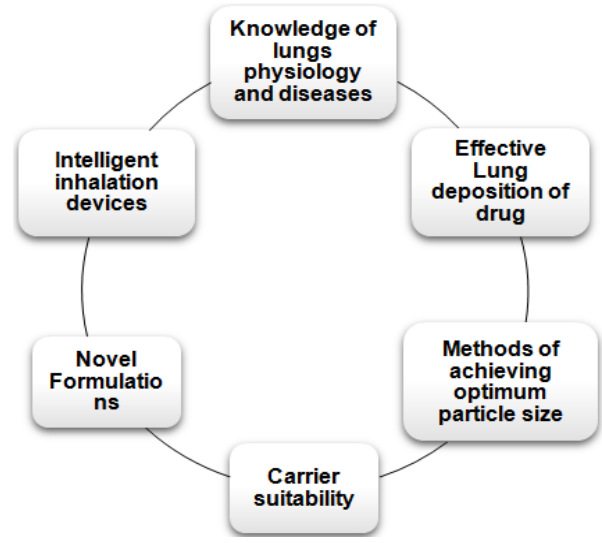

Fig. 2: The factors affecting efficient PDD of NPs
The characteristics of lungs like "aerosol filter" can be utilized for targeting the drug particles with particular attributes to the lungs [48] and also the vast surface area of lungs mucosa helps in systemic absorption of drugs preventing the first pass metabolism [47] and improved bioavailability of drugs.

These merits make the PDD an ideal drug delivery route for the treatment of lung infections like TB. Also, the bacilli of TB infects the AMs [51] and therefore, it is significant to not only deliver a drug to the lungs but also to get the drug particles phagocytized by AMs. The successful inhalation therapy with efficacy and safety in drug delivery of a drug depends on the thorough understanding of designed drug delivery system. The factors affecting the efficient PDD of NPs was shown in fig. 2.

\section{Polymer-based nanosystems for the pulmonary delivery of ATDs}

The use of nanosystems for PDD has shown desirable results in the drug delivery system due to various merits as described earlier. The nanocarriers like lipid-based, liposome and polymer based are common in preparing inhalable carrier system for PDD. Some carrier systems like nanocrystals, magnetic NPs, gold NPs and effervescent NPs are currently in research to study the effect on AMs on delivery. Fig. 3 highlighted the different inhalable nanocarrier systems for ATDs. However, Polymeric NPs are used widely for the drug delivery, and their potentials have been tested for the PDD. Polymeric NPs have shown to be efficient carrier system for PDD as they possess properties like carrier suitability with the drugs, tissue or cell targeting in the lungs, protection from therapeutic degradation, sustained release mechanism, aerosol transformability, less toxicity and stability during the aerosolization [54]. Various therapeutics like antiasthmatic agents, antineoplastic agents, antidiabetic agents, antifungal agents, antimicrobial agents, antiosteoporosis agents, antiparathyroid agents, pulmonary hypertension drugs and ATDs have been formulated into polymeric NPs for PDD. The sustained release characteristics of Polymeric NPs helps in achieving improved bioavailability of ATDs with fewer side effects and decreased the frequency of dosing. These factors result in patient compliance by resolving the problems of non-adherence to prescribed therapy of TB [6]. The toxicity profile of the polymeric NPs allows it for the repeated ATDs administration as required in the treatment of MDR-TB. Various inhalable ATDs loaded polymeric NPs along with their methods of preparation and devices used for PDD is given in table 1.

Table 1: Inhalable polymeric nanoparticles containing ATDs with their method of preparation and device for pulmonary delivery

\begin{tabular}{llllll}
\hline S. No. & Drug & Nanocarrier & Method of preparation & Device & References \\
\hline 1. & CLR & PLGA & ESE & DPI, Cyclohaler® & {$[61]$} \\
2. & INH, PZA and RIF & PLGA & DESE & Compressor-nebulizer (MedelAerofamily) & {$[57,58]$} \\
3. & INH, PZA and RIF & Alginate & IG & Compressor-nebulizer (MedelAerofamily) & {$[55]$} \\
4. & INH & Chitosan & IG & DPI, Cyclohaler & {$[65]$} \\
5. & RIF & PLGA & SE, SD & Intratracheal insufflation & {$[12]$} \\
& & & Novel SD & Dry powder Insufflator"DP-4 & {$[60]$} \\
6. & LVX & PCL, PLGA & ESE & - & {$[61]$} \\
\hline
\end{tabular}

Note: ESE-Emulsification solvent evaporation, DESE-Double emulsification solvent evaporation, IG-Ionic gelation, SE-Solvent evaporation, SD-Spray drying, NP-Nanoprecipitation.

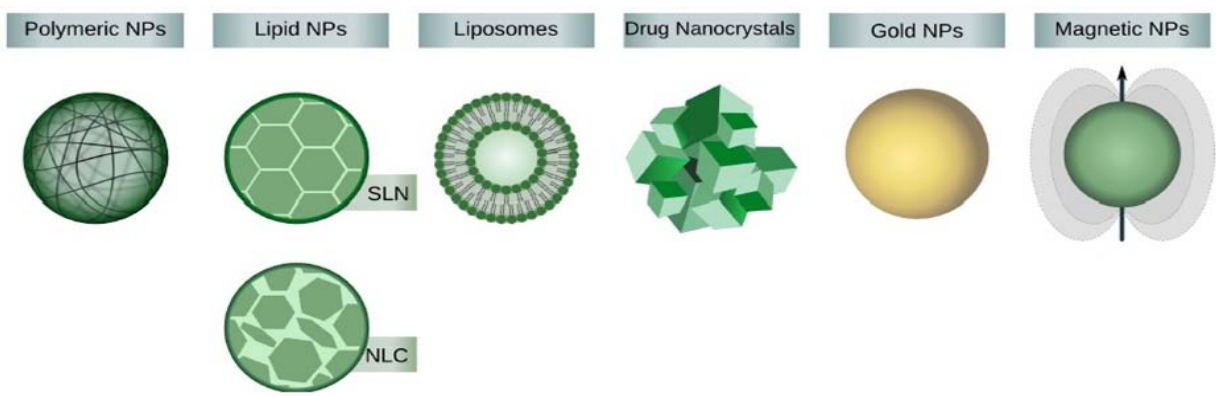

Fig. 3: Different novel nanocarrier systems containing ATDs intended for the pulmonary delivery [23] 
Polymeric NPs containing ATDs were prepared from synthetic and natural, biodegradable polymers such as alginate, PLGA, chitosan and gelatin [53]. These ATDs loaded polymeric nanocarriers are explained in detail as stated below:-

\section{Alginate based NPs containing ATDs}

Alginate is an approved polymer by US FDA for oral route of drug delivery and has been used clinically for the reflux esophagitis. The characteristics of alginate make it a desirable carrier for drug delivery. These properties like adhesiveness with epithelium of intestine, organic solvent free process in a mild room temperature, highly aqueous surrounding in the matrix, high diffusion rates of particle due to high gel porosity, control of porosity with polyelectrolytic interaction, and biodegradability of the polymeric system in the normal physiological makes the alginate an ideal carrier for the controlled release of various therapeutics and drugs including ATDs. The ATDs like INH, RIF, and PZA were loaded into alginate nanocarriers, and this formulation showed desirable aerodynamic diameter and high drug encapsulation efficiency. Also, these NPs showed better bioavailability than the free drugs [55].

\section{PLGA based NPs containing ATDs}

PLGA NPs are the most common polymeric nanoparticulate system containing ATDs. A number of inhalable nanoformulation based on PLGA nanocarriers system for ATDs delivery have been reported earlier. Sung et al. prepared a sustained release formulation of PLGA nanocarriers loaded with RIF for lung delivery [40]. Jain et al. [56] demonstrated that the conjugation of PLGA NPs with lactose has better results due to the active targeting of AMs by lactose. The three first line ATDs namely INH, RIF and PZA have been incorporated into PLGA NPs and the formulation was administered by the oral and inhalable route of drug delivery $[32,33]$. The presence of drugs in plasma was reported for several days, and the inhaled formulation of PLGA NPs containing ATDs showed the equal therapeutic results of daily doses of the oral free drug [57]. Later the same group of researchers coated these nanocarriers with wheat gum agglutinin. The conjugated NPs showed an increase in drug residence in plasma for a number of days [58]. The inhalable dry powder formulations of rifampicin in PLGA nanocarriers have also been reported $[12,59,60]$. A Nano in a microsystem in an inhaled dry powder form containing Clarithromycin (CLR) loaded on PLGA NPs [61]. Cheow et al. [62] prepared the PLGA NPs loaded with Levofloxacin (LVX) and investigated the effect of the antibiotic release profiles of these LVX loaded polymeric nanoparticles on their antibacterial efficacy.

\section{Chitosan-based NPs containing ATDs}

Chitosan possesses certain significant characteristics such as mucoadhesives, enhancement of macromolecules permeation through epithelia, low toxicity, biodegradability and biocompatibility [63]. Due to such useful properties, this polymer has shown desirable pharmaceutical applications. Currently, the cross-linked Chitosan nanocarriers were used with pMDI [64] and this approach can also be used for local therapy of lung infection like TB disease. INH had been loaded into Chitosan nanocarriers and this formulation showed slow and sustained release for $6 \mathrm{~d}$ with initial burst release [65].

\section{Gelatin based NPs containing ATDs}

Gelatin nanocarriers loaded with INH was prepared. The nanocarriers were conjugated with mannose for active targeting in the lungs. The mannosylated nanocarriers showed higher accumulation in the lungs, making them suitable for active targeting of ATDs by the inhalation route of drug delivery [66].

\section{Other polymer based NPs containing ATDs}

LVX loaded Polycaprolactone (PCL) NPs were prepared and characterized [62]. The two types of poly-(ethylene oxide)-block diastearoylphosphatidyl-ethanolamine polymers loaded with RIF were prepared by Abdulla et al. for delivery by inhalation route prepared four different formulations of nanocarriers out of which three were polymeric nanocarriers [67]. The chitosan, gelatin, and albumin nanocarriers were used for loading the drugs and their drug release kinetics was studied. From these polymers, the albumin and chitosan showed better incorporation of drug and sustained release.

\section{Toxicological studies of NPs containing ATDs}

The potential toxicity of the nanomedicine containing ATDs have to be considered and by thorough research and clinical studies, the safety and efficacy of such NDDS must be understood. In a case of Polymeric NPs intended for TB therapy, the polymers used in the composition of such nanocarriers must have clinical approval and safety.

\section{Current scenario of nanosystem containing ATDs}

The conventional ATDs are formulated in the form of capsules and tablets and require a daily dosing. The amount of drug used in such delivery system is high as compared to novel nanocarrier systems. Since a very suboptimal concentration reach the target site of infection through the systemic circulation. The lengthy treatment period with such high concentration of drug leads to some side effects which causes patient non-compliance. The nanosystem containing ATDs have shown desirable results in the animal models for the treatment of TB [68]. However, precise targeting in the lungs still being unmet due to the regional uptake of drugs in the airways with the alveolar region [69]. Still the possibility of dosing the nanosystem containing ATDs weekly or fortnightly have been a key of interest in the nanotechnological research for the TB.

\section{CONCLUSION}

The prevalence of drug-resistant TB has been increased in recent times despite the measures taken by concerned bodies and authorities all around the globe. The conventional chemotherapy of TB has suffered from limitations like a lengthy course of treatment and drugs induce side effects. These limitations result in the development of the resistant strain of TB. Also, the ill-planned therapy is responsible for the drug resistant. The MDR and XDR TB required high doses of second-line ATDs for their lengthy treatment period while these second-line ATDs are more toxic and costly as compared to the first-line drugs. These limitations paved the way for further resistance in the TB strain and the XDR and TDR strain may prove to be deadliest in a TB epidemic with very limited scope for the treatment. The research in the development of new drugs for the drug susceptible and resistant TB is very slow, and the requirements of clinical safety data of this new chemical entity will take a long period of time. Therefore the strategy of developing a novel controlled release polymeric nanoparticulate system containing existing ATDs may help in overcoming the drawbacks associated with the conventional drug delivery system. As these novel polymeric carriers reduce the dose, frequency of dosing and side effects which help in achieving patient compliance. PDD makes it possible to deposit site-specific drugs at high concentrations within the diseased lungs, thereby reducing the overall dose of a drug to patients which in turn reduces systemic side effects. Thus applying nanotechnology together with PDD results in improved patient compliance with a higher bioavailability of ATDs and reduced frequency of dosing thereby it can help in improving patient compliance in the management of drug susceptible and resistant TB. However polymers toxicological studies need to be performed for estimating their safety in clinical use. Therefore, thorough research and commitment by concerned bodies and authorities all over the globe will give rise to less complex, shorter, more effective and less toxic therapy for TB disease in the future.

\section{CONFLICT OF INTERESTS}

Authors declare no conflict of interests.

\section{REFERENCES}

1. Dye C, Watt CJ, Bleed DM, Hosseini SM, Raviglione MC. Evolution of tuberculosis control and prospects for reducing tuberculosis incidence, prevalence, and deaths globally. J Am Med Assoc 2005;293:2767-75.

2. Zumla A, Raviglione M, Hafner R, Von Reyn CF. Tuberculosis. N Engl J Med 2013;368:745-55.

3. Meena L, Rajni. Survival mechanisms of pathogenic mycobacterium tuberculosis H37Rv. FEBS J 2010;277:2416-27. 
4. Sarkar S, Suresh MR. An overview of tuberculosis chemotherapy-a literature review. J Pharm Pharm Sci 2011;14:148-61.

5. Zhang $\mathrm{Y}$, Yew $\mathrm{W}$. Mechanisms of drug resistance in mycobacterium tuberculosis. Int J Tuberculosis Lung Disease 2009;13:1320-30.

6. Kumar G, Malhotra S, Shafiq N, Pandhi P, Khuller G, Sharma S. In vitro physicochemical characterization and short-term in vivo tolerability study of ethionamide loaded PLGA nanoparticles: potentially effective agent for multidrugresistant tuberculosis. J Microencapsul 2011;28:717-28.

7. O'Hara P, Hickey AJ. Respirable PLGA microspheres containing rifampicin for the treatment of tuberculosis: manufacture and characterization. Pharm Res 2000;17:955-61.

8. Suarez S, O'Hara P, Kazantseva M, Newcomer CE, Hopfer R, McMurray DN, et al. Respirable PLGA microspheres containing rifampicin for the treatment of tuberculosis: screening in an infectious disease model. Pharm Res 2001;18:1315-9.

9. Sharma R, Saxena D, Dwivedi AK, Misra A. Inhalable microparticles containing drug combinations to target al. veolar macrophages for the treatment of pulmonary tuberculosis. Pharm Res 2001;18:1405-10.

10. Vyas S, Kannan M, Jain S, Mishra V, Singh P. Design of liposomal aerosols for improved delivery of rifampicin to alveolar macrophages. Int J Pharm 2004;269:37-49.

11. Tewes F, Brillault J, Couet W, Olivier J. Formulation of rifampicin-cyclodextrin complexes for lung nebulization. J Controlled Release 2008;129:93-9.

12. Sung J, Padilla D, Garcia-Contreras L, Ver Berkmoes J, Durbin D, Peloquin C, et al. Formulation and pharmacokinetics of selfassembled rifampicin nanoparticle systems for pulmonary delivery. Pharm Res 2009;26:1847-55.

13. Pandey R. Antitubercular inhaled therapy: opportunities, progress, and challenges. J Antimicrob Chemother 2005;55:430-5.

14. Gupta P, Garg T, Tanmay M, Arora S. Polymeric drug delivery systems: role in P-gp efflux system inhibition. Crit Rev Ther Drug Carrier Syst 2015;32:247-75.

15. Armstrong JA, Hart PD. The response of cultured macrophages to mycobacterium tuberculosis, with observations on fusion of lysosomes with phagosomes. J Exp Med 1971;134:713-40.

16. Aderem A, Underhill D. Mechanisms of phagocytosis in macrophages. Annu Rev Immunol 1999;17:593-623.

17. Pethe K, Swenson D, Alonso S, Anderson J, Wang C, Russell D. Isolation of mycobacterium tuberculosis mutants defective in the arrest of phagosome maturation. Proc Natl Acad Sci USA 2004;101:13642-7.

18. Krishnan N, Robertson B, Thwaites G. The mechanisms and consequences of the extra-pulmonary dissemination of mycobacterium tuberculosis. Tuberculosis 2010;90:361-6.

19. Singh S, Mariappan T, Shankar R, Sarda N, Singh B. A critical review of the probable reasons for the poor variable bioavailability of rifampicin from anti-tubercular fixed-dose combination (FDC) products, and the likely solutions to the problem. Int J Pharm 2001;228:5-17.

20. Panchagnula R, Agrawal S, Ashokraj Y, Varma M, Sateesh K, Bhardwaj V, et al. Fixed-dose combinations for tuberculosis: lessons learned from clinical, formulation and regulatory perspective. Methods Find Exp Clin Pharmacol 2004;26:703.

21. Addington $\mathrm{W}$. Patient compliance: the most serious remaining problem in control of tuberculosis in the United States. Chest 1979;76 Suppl 6:741-3.

22. Bhutani H, Mariappan TT, Singh S. The physical and chemical stability of anti-tuberculosis fixed-dose combination products under accelerated climatic conditions. Int J Tuberculosis Lung Disease 2004;8:1073-80.

23. Ribeiro D. Nanocarrier systems for the delivery of antitubercular drugs. Master Thesis, University of Porto, Porto; 2013.

24. PDO Davies. Drug-resistant tuberculosis. J R Soc Med 2001;94:261-3.

25. Yashodhara B, Huat CB, Naik LN, Umakanth S, Hande M, Pappachan JM. Multidrug and extensively drug-resistant tuberculosis from a general practice perspective. Infect Drug Resist 2010;3:115-22.

26. Sun Z, Zhang J, Song H, Zhang X, Li Y, Tian M, et al. Concomitant increases in spectrum and level of drug resistance in mycobacterium tuberculosis isolates. Int J Tuberculosis Lung Disease 2010;14:1436-41.

27. Caminero J, Sotgiu G, Zumla A, Migliori G. Best drug treatment for multidrug-resistant and extensively drug-resistant tuberculosis. Lancet Infect Dis 2010;10:621-9.

28. Gelperina S, Kisich K, Iseman M, Heifets L. The potential advantages of nanoparticle drug delivery systems in chemotherapy of tuberculosis. Am J Respir Crit Care Med 2005;172:1487-90.

29. Faustini A, Hall AJ, Perucci CA. Risk factors for Multi-drug resistant tuberculosis in Europe: a systematic review. Thorax 2006;61:158-63.

30. Grosset JH, Singer TG, Bishai WR. New drugs for the treatment of tuberculosis: hope and reality. Int J Tuberculosis Lung Disease 2012;16:1005-14.

31. Dutt M, Khuller G. Therapeutic efficacy of Poly(DL-Lactide-CoGlycolide)-encapsulated antitubercular drugs against mycobacterium tuberculosis infection induced in mice. Antimicrob Agents Chemother 2001;45:363-6.

32. Sharma A, Pandey R, Sharma S, Khuller G. Chemotherapeutic efficacy of poly (dl-lactide-co-glycolide) nanoparticle encapsulated antitubercular drugs at sub-therapeutic dose against experimental tuberculosis. Int J Antimicrob Agents 2004;24:599-604.

33. Pandey R, Zahoor A, Sharma S, Khuller G. Nanoparticleencapsulated antitubercular drugs as a potential oral drug delivery system against murine tuberculosis. Tuberculosis 2003;83:373-8

34. Jassal M, Bishai WR. Extensively drug-resistant tuberculosis. Lancet Infect Dis 2009; 9:19-30.

35. Lopes E, Pohlman AR, Bassani V, Guterres SS. Polymeric colloidal systems containing ethionamide: preparation and physicochemical characterization. Pharmazie 2000;55:527-30.

36. Ahmad Z, Pandey R, Sharma S, Khuller G. Novel chemotherapy for tuberculosis: the chemotherapeutic potential of econazole and moxifloxacin-loaded PLG nanoparticles. Int J Antimicrob Agents 2008;31:142-6.

37. Kisich KO, Gelperina S, Higgins MP, Wilson S, Shipulo E, Oganesyan E, et al. Encapsulation of moxifloxacin within poly (butyl cyanoacrylate) nanoparticles enhances efficacy against intracellular mycobacterium tuberculosis. Int J Pharm 2007;345(1, Suppl 2):154-62.

38. Ahmad Z, Sharma S, Khuller G. In vitro and ex vivo antimycobacterial potential of azole drugs against mycobacterium tuberculosis H 37 Rv. FEMS Microbiol Lett 2005;251:19-22.

39. Ahmad Z, Sharma S, Khuller G. Azole antifungals as novel chemotherapeutic agents against murine tuberculosis. FEMS Microbiol Lett 2006;261:181-6.

40. Ahmad Z, Sharma S, Khuller G, Singh P, Faujdar J, Katoch V. Antimycobacterial activity of econazole against multidrugresistant strains of mycobacterium tuberculosis. Int J Antimicrob Agents 2006;28:543-4.

41. Pandey R, Khuller GK. Nanoparticle-based oral drug delivery system for an injectable antibiotic-streptomycin. Evaluation in a murine tuberculosis model. Chemotherapy 2007;53:437-41.

42. Irache JM, Merodio M, Arnedo A, Camapanero MA, Mirshahi M, Espuelas S. Albumin nanoparticles for the intravitreal delivery of anti cytomegaloviral drugs. Mini Rev Med Chem 2005;5:293305.

43. Gill S, Löbenberg $\mathrm{R}, \mathrm{Ku} \mathrm{T}$, Azarmi S, Roa W, Prenner E. Nanoparticles: characteristics, mechanisms of action, and toxicity in pulmonary drug delivery a review. J Biomed Nanotechnol 2007;3:107-19.

44. Gao L, Liu G, Ma J, Wang X, Zhou L, Li X. Drug nanocrystals: in vivo performances. J Controlled Release 2012;160:418-30.

45. Shegokar R, Al Shaal L, Mitri K. Present status of nanoparticle research for treatment of tuberculosis. J Pharm Pharm Sci 2011;14:100-16. 
46. Singh J, Garg T, Rath G, Goyal AK. Advances in nanotechnologybased carrier systems for targeted delivery of bioactive drug molecules with special emphasis on immunotherapy in drug resistant tuberculosis-a critical review. Drug Delivery 2015;11:1-23.

47. Misra A, Hickey A, Rossi C, Borchard G, Terada H, Makino K, et al. Inhaled drug therapy for treatment of tuberculosis. Tuberculosis 2011;91:71-81.

48. Sethi T, Agrawal A. Structure and function of the tuberculous lung: considerations for inhaled therapies. Tuberculosis 2011;91:67-70.

49. Muttil P, Wang C, Hickey A. Inhaled drug delivery for tuberculosis therapy. Pharm Res 2009;26:2401-16.

50. Amani A, Amini M, Ali H, York P. Alternatives to conventional suspensions for pulmonary drug delivery by nebulizers: a review. J Pharm Sci 2011;100:4563-70.

51. Hokey D, Misra A. Aerosol vaccines for tuberculosis: a fine line between protection and pathology. Tuberculosis 2011;91:82-5.

52. Willis L, Hayes D, Mansour H. Therapeutic liposomal dry powder inhalation aerosols for targeted lung delivery. Lung 2012;190:251-62

53. Kaur G, Narang R, Rath G, Goyal A. Advances in pulmonary delivery of nanoparticles. Artif Cells Blood Substitutes Immobilization Biotechnol 2012;40:75-96.

54. Beck-Broichsitter M, Kleimann P, Gessler T, Seeger W, Kissel T, Schmehl T. Nebulization performance of biodegradable sildenafil-loaded nanoparticles using the Aeroneb® pro: formulation aspects and nanoparticle stability to nebulization. Int J Pharm 2012;422:398-408.

55. Zahoor A, Sharma S, Khuller G. Inhalable alginate nanoparticles as antitubercular drug carriers against experimental tuberculosis. Int J Antimicrob Agents 2005;26:298-303.

56. Jain SK, Gupta Y, Ramalingam L, Jain A, Jain A, Khare P, et al. Lactose-conjugated PLGA nanoparticles for enhanced delivery of rifampicin to the lung for effective treatment of pulmonary tuberculosis. PDA J Pharm Sci Technol 2010;64:278-87.

57. Pandey R. Poly (DL-lactide-co-glycolide) nanoparticle-based inhalable sustained drug delivery system for experimental tuberculosis. J Antimicrob Chemother 2003;52:981-6.

58. Sharma A. Lectin-functionalized poly (lactide-co-glycolide) nanoparticles as Oral/Aerosolized antitubercular drug carriers for treatment of tuberculosis. J Antimicrob Chemother 2004;54:761-6.

59. Tomoda K, Ohkoshi T, Kawai Y, Nishiwaki M, Nakajima T, Makino K. Preparation and properties of inhalable nanocomposite particles: effects of the temperature at a spray dryer inlet upon the properties of particles. Colloids Surf B 2008;61:138-44.

60. Ohashi K, Kabasawa T, Ozeki T, Okada H. One-step preparation of rifampicin/Poly (lactic-co-glycolic acid) nanoparticlecontaining mannitol microspheres using a four-fluid nozzle spray drier for inhalation therapy of tuberculosis. J Controlled Release 2009;135:19-24.

61. Moghaddam P, Ramezani V, Esfandi E, Vatanara A, NabiMeibodi M, Darabi M, et al. Development of a nano-micro carrier system for sustained pulmonary delivery of clarithromycin. Powder Technol 2013;239:478-83.

62. Cheow W, Chang MW, Hadinoto K. Antibacterial efficacy of inhalable levofloxacin-loaded polymeric nanoparticles against E. coli biofilm cells: the effect of release antibiotic profile. Pharm Res 2010;27:1597-609.

63. Grenha A, Seijo B, Remuñán-López C. Microencapsulated chitosan nanoparticles for lung protein delivery. Eur J Pharm Sci 2005;25:427-37.

64. Sharma K, Somavarapu S, Colombani A, Govind N, Taylor K. Crosslinked chitosan nanoparticle formulations for delivery from pressurized metered dose inhalers. Eur J Pharm Biopharm 2012;81:74-81.

65. Pourshahab P, Gilani K, Moazeni E, Eslahi H, Fazeli M, Jamalifar H. Preparation and characterization of spray dried inhalable powders containing chitosan nanoparticles for pulmonary delivery of isoniazid. J Microencapsul 2011; 28:605-13.

66. Saraogi G, Sharma B, Joshi B, Gupta P, Gupta UD, Jain NK, et al. Mannosylated gelatin nanoparticles bearing isoniazid for effective management of tuberculosis. J Drug Target 2011;19:219-27.

67. Abdulla J, Tan Y, Darwis Y. Rehydrated lyophilized rifampicinloaded mPEG-DSPE formulations for nebulization. AAPS PharmSciTech 2010;11:663-71.

68. Kurmi B, Kayat J, Gajbhiye V, Tekade R, Jain N. Micro-and nanocarrier-mediated lung targeting. Expert Opin Drug Delivery 2010;7:781-94.

69. Weers J, Bell J, Chan H, Cipolla D, Dunbar C, Hickey AJ, et al. Pulmonary formulations: what remains to be done? J Aerosol Med Pulm Drug Delivery 2010;23 Suppl 2:S5-23.

\section{How to cite this article}

- Jessy Shaji, Mahmood Shaikh. Drug-resistant tuberculosis: recent approach in polymer based nanomedicine. Int J Pharm Pharm Sci 2016;8(10):1-6. 